\title{
Sur les surfaces lorentziennes compactes sans points conjugués
}

\author{
CHRISTOPHE BAVARD \\ PIERRE MOUNOUD
}

\begin{abstract}
Nous prouvons l'existence de métriques sans points conjugués dans toute composante connexe de l'espace des métriques lorentziennes du tore ou de la bouteille de Klein. En particulier, l'existence de tores lorentziens sans points conjugués non plats contraste avec la situation riemannienne (théorème de Hopf).

We prove the existence of metrics without conjugate points in any connected component of the space of Lorentzian metrics on the torus or on the Klein bottle. In particular, the existence of nonflat Lorentzian tori without conjugate points contrasts with the Riemannian case (the Hopf Theorem).
\end{abstract}

$53 \mathrm{C} 50$

\section{Introduction}

D’après le théorème de Hopf [10] (généralisé en toute dimension dans Burago et Ivanov [5]), les tores riemanniens sans points conjugués sont nécessairement plats. En géométrie lorentzienne, le résultat se rapprochant le plus de ce théorème a été obtenu par Andersson, Dahl et Howard [1] (voir aussi Gutiérrez, Palomo et Romero [8, page 373]), où il est montré que toute déformation sans points conjugués et à support compact de la métrique lorentzienne plate standard du plan reste plate; l'analogue riemannien de ce phénomène résulte du théorème de Hopf; voir Green et Gulliver [7].

Même si les ingrédients essentiels de la preuve du théorème de Hopf ne subsistent plus dans le contexte lorentzien, aucun procédé permettant de produire des tores lorentziens sans points conjugués et non plats ne semble connu. Ainsi, la question de l'existence de telles surfaces se pose naturellement. Nous établissons ici le résultat suivant.

Théorème 1 Toute composante connexe de l'espace des métriques lorentziennes sur le tore de dimension 2 ou sur la bouteille de Klein contient des métriques sans points conjugués. 
Il faut noter que les seules surfaces compactes portant des métriques lorentziennes sont le tore et la bouteille de Klein. Dans les deux cas, l'espace des métriques lorentziennes possède une infinité de composantes connexes et l'action du groupe des difféomorphismes sur ces composantes admet une infinité d'orbites. De plus, les métriques plates se situent toutes (au signe près) dans la même composante. Les surfaces sans points conjugués que nous construisons ne sont pas géodésiquement complètes. L'apparition de métriques non complètes est inévitable puisque toutes les métriques complètes appartiennent - au signe près - à une même composante connexe, celle des métriques plates (c'est une conséquence directe de Carrière et Rozoy [6]). Nous laissons ouverte la question de l'existence de tores non plats, sans points conjugués et complets.

Enfin, la situation du point de vue de l'espace des métriques lorentziennes mérite d'être précisée. Nous observons que les métriques sans points conjugués forment un fermé. Ce fermé rencontre toutes les composantes connexes (théorème 1) et nous vérifions que nos exemples sont situés au bord de cet ensemble (section 2.1).

Les exemples présentés dans ce travail se déduisent (par des constructions de revêtements et de quotients) des tores de Clifton-Pohl. Ces tores possèdent par définition un revêtement cyclique commun, que nous appellerons ici "plan de Clifton-Pohl", voir section 1.2. Le point clé est le suivant.

Théorème 2 Le plan de Clifton-Pohl n'a pas de points conjugués.

La preuve de ce résultat s'appuie sur une extension remarquable du plan de Clifton-Pohl introduite par Boubel, Mounoud et Tarquini [2]. Il s'agit d'une surface doublement périodique qui contient le plan de Clifton-Pohl comme ouvert fondamental (à indice 2 près, voir section 1.3). Ce "plan étendu" possède des points conjugués; nous montrons qu'il est géodésiquement complet, nous décrivons l'allure de ses géodésiques (section 1.6) et nous déterminons ses champs de Jacobi à l'aide des fonctions elliptiques (section 1.4). Une étude détaillée de ces champs nous permet de prouver le théorème en exhibant, sur toute géodésique, un champ de Jacobi ne s'annulant pas dans le plan de Clifton-Pohl.

\section{Sur la métrique de Clifton-Pohl}

\subsection{Préliminaire}

Considérons une surface lorentzienne lisse, orientée et munie d'un champ de Killing $K$, c'est-à-dire un champ de vecteurs $K$ vérifiant

$$
\nabla_{X} K \cdot Y+X \cdot \nabla_{Y} K=0
$$


pour tout couple $(X, Y)$ de champs de vecteurs. Soit $\gamma$ une géodésique qui n'est pas de type lumière. Notons $T$ la vitesse "unitaire" de $\gamma$ et $N$ le vecteur "unitaire" directement orthogonal à $T$ :

$$
T \cdot T=\epsilon, \quad N \cdot N=-\epsilon,
$$

avec $\epsilon=1$ (respectivement $\epsilon=-1$ ) si $\gamma$ est de type espace (respectivement temps). Pour abréger, l'entier $\epsilon$ sera appelé le type de $\gamma$. Soit $R$ le tenseur de Riemann. Comme son flot est isométrique, le champ $K$ vérifie l'équation de Jacobi $\nabla_{T} \nabla_{T} K=R(T, K) T$ le long de $\gamma$. Posons

$$
K=C T+\beta N
$$

Alors $C=\epsilon K \cdot T$ est une constante d'après (1) (Lemme de Clairaut et Noether) et $\beta=-\epsilon K \cdot N$. L'équation de Jacobi s'écrit $\beta^{\prime \prime} N=\beta R(T, N) T$, d'où

$$
\beta^{\prime \prime}+\epsilon \kappa \beta=0
$$

où $\kappa$ est la courbure. Cette relation peut également se lire comme l'expression de la courbure $\kappa=-\epsilon \beta^{\prime \prime} / \beta$ aux points où $\beta \neq 0$.

Lemme 1.1 Soit $\gamma$ une géodésique de type $\epsilon= \pm 1$ et soit $\beta=-\epsilon K \cdot N$. Alors la quantité $\epsilon \beta^{\prime}(t)$ ne dépend que du point $\gamma(t)$ et on a la relation

$$
\beta^{\prime 2} K \cdot K+\nabla_{K} K \cdot \nabla_{K} K=0
$$

Preuve Observons d'abord qu'un plan lorentzien (au sens de l'algèbre linéaire) possède deux involutions anti-isométriques naturelles: les réflexions fixant les points d'une droite isotrope $\Delta$ et inversant l'autre. Si le plan est orienté, on peut privilégier l'une des deux, par exemple en prenant pour $\Delta$ la première droite (dans l'ordre de l'orientation) bordant le cône négatif. Cela étant, déterminons $\nabla_{K} K$. On a $\nabla_{T} K=\beta^{\prime} N$, puis $\nabla_{N} K=\beta^{\prime} T$ grâce à (1). Par suite

$$
\nabla_{K} K=\beta^{\prime}(C N+\beta T)=\epsilon \beta^{\prime} K^{*},
$$

où $K^{*}$ désigne l'image de $K$ par l'involution anti-isométrique privilégiée du fibré tangent. Si $\gamma(t)$ n'est pas un zéro de $K$, cette relation permet de conclure. En un zéro de $K$, une étude locale montre que $\epsilon \beta^{\prime}$ est la valeur propre de la partie linéaire de $K$ correspondant à $\Delta$.

Lemme 1.2 Soit $\gamma$ une géodésique sur une surface. On suppose qu'il existe un champ de Jacobi normal le long de $\gamma$ qui ne s'annule pas. Alors la géodésique $\gamma$ n'a pas de points conjugués. 
Preuve C'est un fait bien connu. Soit $f_{0}$ une solution qui ne s'annule pas de l'équation de Jacobi $f^{\prime \prime}+\kappa f=0$ (les fonctions sont à valeurs réelles). Alors pour toute solution $f$ on a $\left(f_{0} f^{\prime}-f f_{0}^{\prime}\right)^{\prime}=f_{0} f^{\prime \prime}-f f_{0}^{\prime \prime}=0$, d'où $\left(f / f_{0}\right)^{\prime}=c / f_{0}^{2}$ avec $c$ constante. Par suite $f / f_{0}$ (et donc $f$ ) s'annule au plus une fois.

Remarque 1.3 Une autre formulation classique de cette propriété est la suivante: étant donné deux solutions indépendantes de l'équation de Jacobi, entre deux zéros de l'une il existe un unique zéro de l'autre. En outre, la réciproque du lemme 1.2 est vraie d'après Hopf [10].

Lemme 1.4 Soit $\gamma$ une géodésique de type temps ou espace sur une surface munie d'un champ de Killing $K$. On suppose que $\gamma$ vérifie l'une des hypothèses suivantes:

- ou bien $C^{2}>\sup |K \cdot K|$ (borne supérieure prise sur la surface),

- ou bien $K$ ne s'annule pas sur $\gamma$ et $\gamma$ est orthogonale à $K(C=0)$.

Alors $\gamma$ n'a pas de points conjugués.

Preuve Dans les deux cas il est évident que la fonction $\beta$ ne peut s'annuler: le lemme 1.2 s'applique.

Enfin, il est utile de noter qu'en dimension 2, une géodésique de type lumière ne peut contenir de points conjugués [14, page 291]. En effet, tout champ de Jacobi le long de $\gamma$ qui s'annule deux fois doit être tangent à $\gamma$ (car perpendiculaire!) et donc nul.

\subsection{Métrique de Clifton-Pohl}

Nous appellerons plan de Clifton-Pohl, noté $\Sigma_{\mathrm{CP}}$, le plan épointé $\mathbf{R}^{2} \backslash\{0\}$ muni de la métrique

$$
g_{\mathrm{CP}}=\frac{2 d x d y}{x^{2}+y^{2}}, \quad(x, y) \in \mathbf{R}^{2} \backslash\{0\} .
$$

L'invariance de cette métrique par les homothéties centrées à l'origine fait du champ radial $K=x \partial_{x}+y \partial_{y}$ un champ de Killing. Le quotient de $\Sigma_{\mathrm{CP}}$ par une homothétie non triviale sera dénommé tore de Clifton-Pohl; il y a donc une famille à un paramètre de tels tores.

Remarquons que la courbure $\kappa$ de $\Sigma_{\mathrm{CP}}$ est liée au carré scalaire de $K$ :

$$
\kappa=-\frac{4 x y}{x^{2}+y^{2}}=-2 K \cdot K=-2 \epsilon\left(C^{2}-\beta^{2}\right),
$$


la dernière égalité valant pour une géodésique donnée $\gamma$, voir (2). L'équation de Jacobi le long de $\gamma$ s'écrit

$$
f^{\prime \prime}+\epsilon \kappa f=f^{\prime \prime}+2\left(\beta^{2}-C^{2}\right) f=0 .
$$

Remarque 1.5 Le signe de $f^{\prime \prime}$ pour une solution $f$ de l'équation de Jacobi coïncide avec celui de $\epsilon x y f$; il ne dépend donc que du type $\epsilon$ (fixé), du quadrant où l'on se place et du signe de $f$.

Lemme 1.6 (localisation des géodésiques) Soit $\gamma$ une géodésique de $\Sigma_{\mathrm{CP}}$. Alors

(i) les coordonnées $x$ et $y$ sont monotones le long de $\gamma$,

(ii) $\gamma$ reste dans un demi-plan déterminé par un axe d'équation $x=0$ ou $y=0$,

(iii) la courbure s'annule au plus une fois le long de $\gamma$.

Preuve (i) Cela résulte de la préservation du type.

(ii) Soit $\gamma$ une géodésique, que l'on peut supposer de type \pm 1 . Si $\gamma$ coupe deux fois (au moins) l'ensemble $x y=0$, on considère deux points d'intersection consécutifs avec $x y=0$, que l'on peut supposer (par symétrie de la connexion de Levi-Civita) sur les demi-axes $y=0, x>0$ et $x=0, y>0$; l'intégrale de Clairaut conduit immédiatement à une contradiction.

(iii) C'est une conséquence directe de (i) et (ii) et du fait que $\kappa$ s'annule si et seulement si $x y=0$.

Dans le plan de Clifton-Pohl, la relation (3) s'écrit

$$
\nabla_{K} K=\frac{x^{2}-y^{2}}{x^{2}+y^{2}} K^{*}
$$

Les seules trajectoires géodésiques du champ de Killing sont donc les demi-diagonales $x^{2}=y^{2}$; elles correspondent aux points critiques de la fonction $K \cdot K$ (c'est une propriété générale) qui coïncident ici avec les extrema de la courbure.

Nous montrerons plus loin (voir section 1.4) que $\beta$ s'exprime au moyen des fonctions elliptiques de Jacobi. Dans un premier temps, il est naturel d'examiner les zéros de $\beta$, c'est-à-dire les zéros des champs de Jacobi induits par $K$.

Lemme 1.7 (sur les zéros de $\beta$ ) Soit $\gamma$ une géodésique (de type temps ou espace) du plan de Clifton-Pohl. Alors

- ou bien $\beta \equiv 0$ et $\gamma$ est portée par une trajectoire de Killing (demi-diagonale $\left.x^{2}=y^{2}\right)$,

- ou bien $\beta$ s'annule au plus une fois. 
Preuve Les zéros de $\beta$ correspondent aux points où $\gamma$ est tangente à $K$. Si $\beta$ est identiquement nulle, alors $\gamma$ est au paramétrage près une trajectoire du champ de Killing. Sinon, supposons que $\beta(a)=\beta(b)=0$ avec $a<b$; alors $K$ a le type de $\gamma$ en $\gamma(a)$ et $\gamma(b)$ et (lemme 1.6) tous les points $\gamma(t)$ pour $a \leq t \leq b$ appartiennent à un même quadrant ouvert du plan $(x, y)$. D'après la remarque 1.5 , la fonction $\beta^{\prime \prime}$ est du signe de $\beta$ sur $] a, b$ [, ce qui est absurde.

Proposition 1.8 (premières géodésiques sans points conjugués) Soit $\gamma$ une géodésique (de type temps ou espace) du plan de Clifton-Pohl avec $|C| \geq 1$ ou $C=0$. Alors $\gamma$ ne contient pas de points conjugués.

Preuve Les cas $C^{2}>1$ et $C=0$ résultent du lemme 1.4. Si $C^{2}=1$ et si $\beta$ s'annule, alors $\gamma$ est tangente à $K$ en un point où $K \cdot K$ est extrémal, c'est-à-dire vérifiant $x^{2}=y^{2}$. Ainsi $\gamma$ est portée par une trajectoire de $K$ et $\beta$ est identiquement nulle. Le long de $\gamma$ la courbure $\kappa$ est donc constante, plus précisément $\epsilon \kappa=-2$. Il est donc clair que l'équation (6) n'a pas de solutions s'annulant deux fois.

Les éventuels points conjugués du plan de Clifton-Pohl sont donc à rechercher sur les géodésiques admettant une unique tangence avec le champ de Killing (c'est-à-dire $\beta$ admet un unique zéro); de plus les champs de Jacobi associés ne peuvent avoir que deux zéros situés de part et d'autre du zéro de $\beta$. Noter par ailleurs que les extrema de $\beta$ sont situés aux intersections de $\gamma$ avec les diagonales $x^{2}=y^{2}$ (comparer (3) et (7)).

\subsection{Extension du plan de Clifton-Pohl}

Dans [2], il est montré que le plan de Clifton-Pohl admet une extension naturelle. Plus précisément, il existe une surface $\widehat{\Sigma}$ doublement périodique dont un pavé fondamental ouvert - à indice 2 près - est isométrique à $\Sigma_{\mathrm{CP}}$. Posons

$$
\Lambda=\left\{\frac{\pi}{2}(k, l) ;(k, l) \in \mathbf{Z}^{2}, k+l \equiv 0(\bmod 2)\right\} .
$$

Les éléments de ce réseau seront considérés indifféremment comme points du plan ou comme translations. Nous appellerons plan étendu, bien qu'il ne s'agisse pas d'un plan au sens strict, la surface $\widehat{\Sigma}=\left(\mathbf{R}^{2} \backslash \Lambda, \widehat{g}\right)$, où $\widehat{g}$ est la métrique définie par

$$
\widehat{g}=\frac{2 d \varphi d \psi}{\cos ^{2} \varphi \sin ^{2} \psi+\sin ^{2} \varphi \cos ^{2} \psi}, \quad(\varphi, \psi) \in \mathbf{R}^{2} \backslash \Lambda .
$$

Soit $U$ l'ouvert formé des points $(\varphi, \psi) \in \widehat{\Sigma}$ tels que $|\varphi|<\pi / 2,|\psi|<\pi / 2$ (carré épointé). Un calcul immédiat montre que l'application $\Psi: U \rightarrow \Sigma_{\mathrm{CP}}$ définie par 
$\Psi(\varphi, \psi)=(\tan \varphi, \tan \psi)$ est une isométrie. Il est par ailleurs clair que le réseau $\Lambda$ agit isométriquement sur $\widehat{\Sigma}$. Ainsi l'ouvert $U$ est isométrique au plan de Clifton-Pohl $\Sigma_{\mathrm{CP}}$ et constitue un domaine fondamental ouvert pour un sous-réseau d'indice 2 de $\Lambda$ (à savoir $\pi \mathbf{Z}^{2}$ ). Noter également que $\widehat{\Sigma}$ n'a qu'une singularité modulo $\Lambda$, celle de $\Sigma_{\mathrm{CP}}$. Dans la suite, nous identifierons l'ouvert $U$ avec $\Sigma_{\mathrm{CP}}$.

Le champ de Killing de $\Sigma_{\text {CP }}$ s'étend en un champ de Killing sur $\widehat{\Sigma}$, encore noté $K$, partout défini dans le plan $(\varphi, \psi)$ et donné par

$$
2 K=\sin (2 \varphi) \partial_{\varphi}+\sin (2 \psi) \partial_{\psi}, \quad(\varphi, \psi) \in \mathbf{R}^{2} .
$$

La relation (5) entre la courbure et le carré du champ de Killing se prolonge évidemment au plan étendu:

(8) $\kappa=-2 K \cdot K=\delta^{-1} \sin (2 \varphi) \sin (2 \psi), \quad$ où $\delta=\cos ^{2} \varphi \sin ^{2} \psi+\sin ^{2} \varphi \cos ^{2} \psi$.

On voit que la courbure s'annule sur les deux familles de droites $\varphi=\pi / 2(\bmod \pi / 2)$ et $\psi=\pi / 2(\bmod \pi / 2)$. Noter par ailleurs que la relation particulière $\kappa=-2 K \cdot K$ montre que la fonction $\beta$ est solution de

$$
\beta^{\prime \prime}+2\left(\beta^{2}-C^{2}\right) \beta=0
$$

Il est immédiat d'expliciter le groupe d'isométries de $\widehat{\Sigma}$. En effet, l'ensemble des points de $\widehat{\Sigma}$ par lesquels passent deux lignes de courbure nulle est stable par $\operatorname{Isom}(\widehat{\Sigma})$; grâce à l'action du réseau $\Lambda$, on se ramène à déterminer le stabilisateur $G$ de l'un de ces points, par exemple $p_{0}=(0, \pi / 2)$. Le morphisme évident de $G$ dans le groupe orthogonal de Lorentz $\boldsymbol{O}(1,1)$ est injectif (car tout point de $\widehat{\Sigma}$ est relié à $p_{0}$ par une géodésique brisée). On constate facilement qu'il est surjectif, la composante neutre de $\boldsymbol{O}(1,1)$ correspondant à la composante neutre $\operatorname{Isom}_{0}(\widehat{\Sigma})$, c'est-à-dire au flot du champ $K$. La projection de $\operatorname{Isom}(\widehat{\Sigma}) \operatorname{sur} \operatorname{Isom}(\widehat{\Sigma}) / \operatorname{Isom}_{0}(\widehat{\Sigma})$ est scindée et on a la suite exacte (également scindée)

$$
0 \longrightarrow \Lambda \longrightarrow \operatorname{Isom}(\widehat{\Sigma}) / \operatorname{Isom}_{0}(\widehat{\Sigma}) \longrightarrow \mathbf{Z} / 2 \mathbf{Z} \times \mathbf{Z} / 2 \mathbf{Z} \longrightarrow 0 .
$$

Ce quotient $\operatorname{Isom}(\widehat{\Sigma}) / \operatorname{Isom}_{0}(\widehat{\Sigma})$ est isomorphe au "groupe de réflexions" associé au carré euclidien (les réflexions par rapports aux diagonales $\varphi \pm \psi=n \pi / 2, n \in \mathbf{Z}$, étant des isométries). Noter que la surface $\widehat{\Sigma}$ admet également des anti-isométries. 


\subsection{Champs de Jacobi du plan étendu}

Proposition 1.9 Soit $\gamma$ une géodésique de type $\epsilon= \pm 1$ du plan étendu. Le long de $\gamma$ la fonction $\beta$ et la courbure $\kappa$ vérifient les équations différentielles suivantes:

$$
\begin{aligned}
\beta^{\prime 2}+\frac{\kappa^{2}}{4}=\beta^{\prime 2}+\left(\beta^{2}-C^{2}\right)^{2} & =1, \\
\kappa^{\prime 2}+2\left(\epsilon \kappa+2 C^{2}\right)\left(\kappa^{2}-4\right) & =0 .
\end{aligned}
$$

Preuve On oriente le plan étendu par $\left(\partial_{\varphi}, \partial_{\psi}\right)$. L'identité (3) donne alors

$$
\epsilon \beta^{\prime}=\delta^{-1}\left(\cos ^{2} \psi-\cos ^{2} \varphi\right),
$$

qui entraîne immédiatement l'équation (10) compte tenu de (8). De la relation (5) on déduit que $\kappa^{\prime}=4 \epsilon \beta \beta^{\prime}$. Par suite

$$
\kappa^{\prime 2}=16 \beta^{2} \beta^{\prime 2}=16\left(\epsilon \kappa / 2+C^{2}\right)\left(1-\kappa^{2} / 4\right) .
$$

Soit $\gamma$ une géodésique définie sur un intervalle $I$ (a priori distinct de $\mathbf{R}$ ). Dans la suite, nous dirons qu'une fonction $f$ définie le long de $\gamma$ se déduit d'une fonction $f_{0}$ définie sur $\mathbf{R}$ s'il existe $t_{0} \in \mathbf{R}$ et $\eta= \pm 1$ tels que $f(t)=f_{0}\left(\eta t+t_{0}\right)$, pour tout $t \in I$. Les équations différentielles (9) et (10) nous permettent d'exprimer les fonctions $\beta$ le long de $\gamma$ à l'aide de fonctions elliptiques de Jacobi sn, cn et $\mathrm{dn}$. On pose également $\mathrm{sd}=\mathrm{sn} / \mathrm{dn}$ et $\mathrm{nd}=1 / \mathrm{dn}$.

Proposition 1.10 (fonctions $\beta$ du plan étendu) Soit $\gamma$ une géodésique de type $\epsilon= \pm 1$. Soit $\beta$ la fonction définie le long de $\gamma \operatorname{par} \beta=-\epsilon K \cdot N$ et soit $C$ la constante donnée par $C=\epsilon K \cdot T$ (voir section 1.1). Au signe près, la fonction $\beta$ se déduit de la fonction $\beta_{0}$ définie sur $\mathbf{R}$ par:

$$
\beta_{0}(t)= \begin{cases}\sqrt{\frac{1-C^{4}}{2}} \operatorname{sd}\left(\sqrt{2} t, \sqrt{\frac{1+C^{2}}{2}}\right) & \text { si } C^{2}<1, \\ \frac{8 e^{\sqrt{2} t}}{8 e^{2 \sqrt{2} t}+1} \text { ou } 0 & \text { si } C^{2}=1, \\ \sqrt{C^{2}-1} \operatorname{nd}\left(\sqrt{C^{2}+1} t, \sqrt{\frac{2}{C^{2}+1}}\right) & \text { si } C^{2}>1 .\end{cases}
$$

Preuve À partir des identités classiques entre les fonctions elliptiques de Jacobi $\mathrm{sn}^{2}+\mathrm{cn}^{2}=1, \quad \mathrm{dn}^{2}+\ell^{2} \mathrm{sn}^{2}=1, \quad \mathrm{sn}^{\prime}=\mathrm{cndn}, \quad \mathrm{cn}^{\prime}=-\mathrm{dn} \mathrm{sn}, \quad \mathrm{dn}^{\prime}=-\ell^{2} \mathrm{cnsn}$, où $\ell$ est "le module", ici $\ell^{2}=\left(1+C^{2}\right) / 2$ si $C^{2}<1$ ou $\ell^{2}=2 /\left(C^{2}+1\right)$ si $C^{2}>1$, on vérifie que les fonctions définies par (13) donnent bien des solutions des équations 
(9) et (10). Pour avoir toutes les solutions il suffit de considérer les fonctions déduites de $\beta_{0}$ sur $\mathbf{R}$ et leurs opposées. La connaissance d'un couple $\left(\gamma\left(t_{0}\right), \gamma^{\prime}\left(t_{0}\right)\right)$ nous donne $\left(\beta\left(t_{0}\right), \beta^{\prime}\left(t_{0}\right)\right)$ via l'identité (12) et permet de déterminer $\beta$.

On s'intéresse maintenant aux géodésiques du plan étendu pouvant posséder des points conjugués. Au vu du lemme 1.4, on peut supposer que $|C|<1$ dans ce qui suit.

Proposition 1.11 (expression de la courbure, $|C|<1$ ) Soit $\gamma$ une géodésique de type $\epsilon= \pm 1$ avec $|C|<1$ et $\beta(0)=0$. Alors, le long de $\gamma$, la courbure $\kappa$ et la fonction $\beta^{2}$ sont données par

$$
\kappa=-2 \epsilon\left(C^{2}+\frac{C^{4}-1}{\wp-2 C^{2} / 3}\right) \quad \text { et } \quad \beta^{2}=\frac{1-C^{4}}{\wp-2 C^{2} / 3},
$$

où $\wp$ est la fonction de Weierstrass de paramètres $g_{2}=\frac{4}{3}\left(C^{4}+3\right)$ et $g_{3}=\frac{8 C^{2}}{27}\left(C^{4}-9\right)$.

Preuve En posant $\kappa=-2 \epsilon\left(w+C^{2} / 3\right)$, l'équation de la courbure (11) se met sous la forme "de Weierstrass"

$$
w^{\prime 2}=4 w^{3}-\frac{4}{3}\left(C^{4}+3\right) w-\frac{8}{27} C^{2}\left(C^{4}-9\right)=4\left(w-e_{1}\right)\left(w-e_{2}\right)\left(w-e_{3}\right),
$$

avec $e_{1}=1-C^{2} / 3, e_{2}=2 C^{2} / 3$ et $e_{3}=-1-C^{2} / 3$. On a donc $w(t)=\wp(t+a)$, où $a$ vérifie $\wp(a)=e_{2}$ (condition $\kappa(0)=-2 \epsilon C^{2}$ ) et $\wp^{\prime}(a)=0$. Par suite (formule d'addition pour $\wp)$ :

$\wp(t+a)=\frac{1}{4}\left(\frac{\wp^{\prime}(t)}{\wp(t)-e_{2}}\right)^{2}-\wp(t)-e_{2}=e_{2}+\frac{\left(e_{2}-e_{1}\right)\left(e_{2}-e_{3}\right)}{\wp(t)-e_{2}}=\frac{2}{3} C^{2}+\frac{C^{4}-1}{\wp(t)-e_{2}}$,

d'où l'expression de $\kappa$. Celle de $\beta^{2}$ résulte maintenant de (5).

D'après la proposition 1.10, les champs de Jacobi le long d'une géodésique de type \pm 1 se déduisent des solutions de l'équation différentielle

$$
f^{\prime \prime}-2\left(C^{2}-\beta_{0}^{2}\right) f=0 .
$$

Proposition 1.12 (solutions de l'équation de Jacobi, $|C|<1$ ) L'espace vectoriel des solutions sur $\mathbf{R}$ de l'équation “de Jacobi” (15) est engendré par les fonctions

$$
\mathfrak{s}(t)=\operatorname{sd}\left(\sqrt{2} t, \sqrt{\left(1+C^{2}\right) / 2}\right) \quad \text { et } \quad \mathfrak{c}(t)=\left(3 \zeta(t)+2 C^{2} t\right) \mathfrak{s}(t),
$$

où $\zeta$ est la fonction zêta de Weierstrass de paramètres $g_{2}=4\left(C^{4}+3\right) / 3$ et $g_{3}=$ $8 C^{2}\left(C^{4}-9\right) / 27$. De plus, la fonction $\mathfrak{c} n$ 'est jamais périodique. 
Preuve Comme $\beta_{0}$ vérifie l'équation (9), la fonction $\mathfrak{s}$ est solution. De plus $\mathfrak{c}=\lambda \mathfrak{s}$ est solution si et seulement si $\lambda^{\prime \prime} \mathfrak{s}+2 \lambda^{\prime} \mathfrak{s}^{\prime}=0$. La deuxième relation de (14) implique $\mathfrak{s}^{2}\left(3 \wp-2 C^{2}\right)=6$, donc $\lambda(t)=3 \zeta(t)+2 C^{2} t$ convient (on rappelle que $\left.\zeta^{\prime}=-\wp\right)$ et le wronskien de $\mathfrak{s}$ et $\mathfrak{c}$ vaut alors -6 . Noter que la fonction $\zeta \mathfrak{s}$ est holomorphe près de l'axe réel.

On définit une fonction méromorphe $Z$ sur $\mathbf{C}$ par $Z(z)=3 \zeta(z)+2 C^{2} z$. Il est bien connu (voir [9]) que la fonction $\zeta$ est quasi-bipériodique, c'est-à-dire il existe deux nombres complexes $\omega_{1}, \omega_{2}$, indépendants sur $\mathbf{R}$, tels que

$$
\zeta\left(z+2 n \omega_{1}+2 m \omega_{2}\right)=\zeta(z)+2 n \zeta\left(\omega_{1}\right)+2 m \zeta\left(\omega_{2}\right),
$$

pour tout $(n, m) \in \mathbf{Z}^{2}$. Comme nos fonctions $\zeta$ sont associées à des fonctions elliptiques de Jacobi, on sait de plus que $\omega_{1}$ et $i \omega_{2}$ sont réels. On en déduit que $Z$ est également quasi-périodique:

$$
Z\left(z+2 n \omega_{1}+2 m \omega_{2}\right)=Z(z)+2 n Z\left(\omega_{1}\right)+2 m Z\left(\omega_{2}\right),
$$

pour tout $(n, m) \in \mathbf{Z}^{2}$. Pour estimer le défaut de périodicité réel (donné par $Z\left(\omega_{1}\right)$ ), on peut donc se placer sur une droite horizontale arbitraire ne passant pas par un pôle de $\zeta$ et intégrer la dérivée de $Z$ sur une période. On sait que pour tout $z \in \mathbf{C}$,

$$
Z^{\prime}(z)=-\frac{6}{\mathfrak{s}^{2}(z)}
$$

Par ailleurs, pour tout $u \in \mathbf{R}$, on a (voir [9, page 47]) $\operatorname{sd}\left(u+\sqrt{2} \omega_{2}\right) \in i \mathbf{R}$. Il en résulte que pour tout $t \in \mathbf{R}, Z^{\prime}\left(t+\omega_{2}\right)$ est un réel positif et que donc $Z\left(\omega_{1}\right)$ est un réel strictement positif. La fonction $Z$ n'est donc jamais périodique et $\mathfrak{c}$ ne l'est jamais non plus.

\subsection{Absence de points conjugués dans le plan de Clifton-Pohl}

Proposition 1.13 Soit $\gamma$ une géodésique de $\widehat{\Sigma}$ de type $\epsilon= \pm 1$ et soit $J$ un champ de Jacobi non trivial le long de $\gamma$. Alors la courbure $\kappa$ s'annule au moins deux fois (multiplicité comprise) sur tout segment de $\gamma$ délimité par deux zéros distincts de $J$.

Preuve Si le champ $J$ s'annule deux fois, on a nécessairement $|C|<1$; de plus (lemme 1.2) la fonction $\beta$ doit s'annuler entre deux zéros consécutifs de $J$. Quitte à reparamétrer $\gamma$, on peut supposer que $\beta(0)=0$. Sur l'intervalle de définition de $\gamma$, on a alors $\kappa(t)=-2 \epsilon\left(C^{2}-\beta_{0}(t)\right)^{2}$ (proposition 1.11) et le champ $J$ est défini par la restriction d'une solution non triviale $f$ de l'équation de Jacobi (15). Si $f$ est proportionnelle à $\mathfrak{s}$, la conclusion est vraie. Sinon, $f$ admet deux zéros consécutifs $t_{1}$ et $t_{2}$ tels que $t_{1}<0<t_{2}$. Soit $t_{0} \geq 0$ le plus petit réel tel que $\beta_{0}\left(t_{0}\right)=|C|$, ou encore 
$\mathfrak{s}\left(t_{0}\right)=\sqrt{2}|C|\left(1-C^{4}\right)^{-1 / 2}$. On a $t_{0}=0$ si et seulement si $C=0$ et dans ce cas le segment $\left[t_{1}, t_{2}\right]$ comprend un zéro de $\mathfrak{s}$ (lemme 1.2), c'est-à-dire un zéro double de la courbure. Nous supposons donc maintenant que $C \neq 0\left(t_{0}>0\right)$. Rappelons que $\omega_{1}$ désigne le quart de période réel de $\mathfrak{s}$ et que $t_{0}<2 \omega_{1}-t_{0}$. Les relations

$$
\mathfrak{s}\left(2 \omega_{1}-t_{0}\right)=\mathfrak{s}\left(t_{0}\right) \quad \text { et } \quad \mathfrak{s}\left(-t_{0}\right)=-\mathfrak{s}\left(t_{0}\right),
$$

signifient que la fonction $C^{2}-\beta_{0}^{2}$ s'annule en $-t_{0}, t_{0}$ et en $2 \omega_{1}-t_{0}$. On peut supposer que l'un au moins des réels $t_{1}$ ou $t_{2}$ appartient à $\left[-t_{0}, t_{0}\right]$ (sinon il n'y a plus rien à prouver) et même, quitte à inverser le sens de parcours de $\gamma$, que $t_{1} \in\left[-t_{0}, t_{0}\right]$. Ainsi, pour montrer que la courbure s'annule deux fois sur $\gamma$ entre les points $\gamma\left(t_{1}\right)$ et $\gamma\left(t_{2}\right)$, il suffit de trouver une solution de l'équation (15) ne s'annulant pas sur ] $-t_{0}, 2 \omega_{1}-t_{0}$ [ (cela entraîne $t_{2} \geq 2 \omega_{1}-t_{0}$ d'après le lemme 1.2).

Soit $f_{0}$ la solution de l'équation de Jacobi donnée par

$$
f_{0}=\mathfrak{s}+\frac{\mathfrak{s}\left(t_{0}\right)}{\mathfrak{c}\left(t_{0}\right)} \mathfrak{c}=\mathfrak{s}\left(1+\frac{Z}{Z\left(t_{0}\right)}\right) .
$$

La fonction $\mathfrak{s}$ est impaire tandis que $\mathfrak{c}$ est paire, donc $f_{0}\left(-t_{0}\right)=0$. On en déduit, en utilisant à nouveau le lemme 1.2 , que $f_{0}$ ne s'annule pas sur $\left.]-t_{0}, 0\right]$.

On sait que $\mathfrak{c}(0)=3 \sqrt{2}>0$. Comme l'expression $C^{2}-\beta_{0}^{2}$ est positive sur $]-t_{0}, t_{0}[$ et que $\mathfrak{c}$ est solution de l'équation de Jacobi, on voit que $\mathfrak{c}$ est convexe sur $]-t_{0}, t_{0}$ [. Comme $\mathfrak{c}^{\prime}(0)=0$ on en déduit que $\mathfrak{c}\left(t_{0}\right)>\mathfrak{c}(0)>0$. On a bien évidemment $f_{0}(0)=$ $\left(\mathfrak{s}\left(t_{0}\right) / \mathfrak{c}\left(t_{0}\right)\right) \mathfrak{c}(0)>0$ et $f_{0}^{\prime}(0)=\mathfrak{s}^{\prime}(0)>0$. L'équation de Jacobi implique alors que $f_{0}$ est convexe sur $]-t_{0}, t_{0}$ [. Par suite $f_{0}\left(t_{0}\right)$ est lui aussi positif. En appliquant encore le lemme 1.2 , on obtient que $f_{0}$ s'annule sur $]-t_{0}, 2 \omega_{1}-t_{0}$ [ si et seulement si $f_{0}\left(2 \omega_{1}-t_{0}\right)$ est négatif.

En utilisant le fait que $Z$ est impaire et quasi-périodique on a

$$
\begin{aligned}
f_{0}\left(2 \omega_{1}-t_{0}\right) & =\mathfrak{s}\left(t_{0}\right)\left(1+\frac{Z\left(2 \omega_{1}-t_{0}\right)}{Z\left(t_{0}\right)}\right) \\
& =\mathfrak{s}\left(t_{0}\right)\left(1+\frac{Z\left(-t_{0}\right)+2 Z\left(\omega_{1}\right)}{Z\left(t_{0}\right)}\right)=2 \mathfrak{s}\left(t_{0}\right) \frac{Z\left(\omega_{1}\right)}{Z\left(t_{0}\right)} .
\end{aligned}
$$

De plus, on sait que $Z\left(t_{0}\right)>0$ et on a vu lors de la preuve de la proposition 1.12 que $Z\left(\omega_{1}\right)>0$. Par suite $f_{0}\left(2 \omega_{1}-t_{0}\right)$ est positif, ce qui montre que $f_{0}$ ne s'annule pas sur $]-t_{0}, 2 \omega_{1}-t_{0}[$ et termine la preuve.

Nous pouvons maintenant conclure la démonstration du théorème 2. Soit $\gamma$ une géodésique du plan de Clifton-Pohl. Si $\gamma$ est de type lumière, elle n'a pas de points conjugués (voir section 1.1). Sinon, d'après le lemme 1.6, la courbure ne peut s'annuler qu'une 
fois le long de $\gamma$. Or, la proposition 1.13 nous dit qu'entre deux points conjugués la courbure doit s'annuler deux fois (le cas $C=0$ est exclus par la proposition 1.8). Il n'y a donc pas de points conjugués sur $\gamma$.

\subsection{Complétude et géodésiques du plan étendu}

Proposition 1.14 Le plan étendu $\widehat{\Sigma}$ est géodésiquement complet.

Preuve Commençons par étudier la complétude des géodésiques de type lumière de $\widehat{\Sigma}$. Soit $\gamma$ une telle géodésique. Si $\gamma$ ne s'accumule pas sur des points de $\Lambda$, elle est préservée par une translation isométrique. Sinon, elle est donnée par $\gamma(t)=$ $\left(\arctan \left(t_{0}+t\right), \pi / 2\right)$ modulo isométrie. Dans les deux cas la géodésique $\gamma$ est complète.

Regardons la complétude des autres géodésiques. Comme il existe des anti-isométries, il nous suffit d'étudier la complétude des géodésiques $\gamma$ de type espace. Les coordonnées $\varphi$ et $\psi$ étant strictement monotones le long de $\gamma$, la géodésique ne peut s'accumuler dans $\widehat{\Sigma}$. Il y a donc deux comportements possibles aux bornes de l'intervalle de définition de $\gamma$ : la géodésique $\gamma$ part à l'infini ou elle va s'accumuler sur un point de $\Lambda$.

Dans le premier cas la courbure s'annule une infinité de fois le long de $\gamma$, autrement dit le carré de la fonction $\beta$ associée à $\gamma$ prend une infinité de fois la valeur $C^{2}$. Or aucune des expressions de $\beta^{2}$ données par la proposition 1.10 ne prend une infinité de fois la valeur $C^{2}$ sur un intervalle borné. On en déduit que si la géodésique $\gamma$ ne s'accumule pas sur un point singulier alors elle est complète.

Dans le deuxième cas, quitte à translater $\gamma$ par un élément de $\Lambda$, on peut supposer que $\gamma$ s'accumule (dans le futur) sur l'origine de $\widehat{\Sigma}$. On peut donc travailler dans le plan de Clifton-Pohl $\Sigma_{\mathrm{CP}}$ (voir (4)). D'après le lemme 1.6, pour $t$ assez grand, le point $\gamma(t)$ appartient à un quart de plan que l'on notera $V$. Modulo isométrie, on choisit $V=\left\{(x, y) \in \Sigma_{\mathrm{CP}} ; x>0, y>0\right\}$. Dans ce cas $x$ et $y$ décroissent le long de $\gamma$ et $C \leq 0$. On a $C \neq 0$, sinon $\gamma$ est contenue dans $\left\{(x, y) \in \Sigma_{\mathrm{CP}} ; x y=\right.$ cte $\left.\neq 0\right\}$ et $\gamma$ ne s'accumule pas sur 0 . Dans les coordonnées $(u, \theta)$ définies par $x=\exp (u) \cos (\theta)$, $y=\exp (u) \sin (\theta)$ la métrique s'écrit:

$$
\left.\sin 2 \theta\left(d u^{2}-d \theta^{2}\right)+2 \cos 2 \theta d u d \theta, \quad(u, \theta) \in \mathbf{R} \times\right] 0, \pi / 2[,
$$

avec bien sûr $K=\partial_{u}$. Si $\beta$ est identiquement nulle alors $\gamma$ est une trajectoire de $K$ et donc $\gamma$ est complète. Sinon, les zéros de $\beta$ sont isolés et quitte à avancer le long de $\gamma$, on peut supposer que $\gamma$ est transverse à $K$, c'est-à-dire que $\theta^{\prime}$ ne s'annule pas. Par hypothèse, $\gamma$ tend vers 0 , c'est-à-dire $u$ tend vers $-\infty$. Si $\gamma$ n'est pas complète, alors forcément $u^{\prime}$ n'est pas bornée. Montrons que ce n'est pas possible. Des intégrales 
premières (à savoir $\gamma^{\prime}(t) \cdot \gamma^{\prime}(t)=1$ et $\gamma^{\prime}(t) \cdot K=C$ ) dans les coordonnées $(u, \theta)$, on déduit

$$
\left\{\begin{array}{l}
u^{\prime} \sin (2 \theta)+\theta^{\prime} \cos (2 \theta)=C \\
\left(\theta^{\prime}\right)^{2}=C^{2}-\sin (2 \theta)
\end{array}\right.
$$

Si $\theta^{\prime}>0$, alors

$$
u^{\prime} \sin (2 \theta)=C\left(1+\cos (2 \theta) \sqrt{1-\sin (2 \theta) / C^{2}}\right)
$$

Comme $\theta$ ne peut pas tendre vers $0, u^{\prime}$ est bornée et $\gamma$ est (semi-)complète. Le cas $\theta^{\prime}<0$ se traite de la même façon.

La complétude et la connaissance des fonctions $\beta$ (proposition 1.10) nous permettent d'une part de préciser l'allure des géodésiques de $\widehat{\Sigma}$ et des tores de Clifton-Pohl (proposition 1.15), d'autre part d'exhiber un ouvert de $\widehat{\Sigma}$ ayant un quotient compact et des points conjugués (remarque 1.17). Appelons axes verticaux (respectivement horizontaux) les droites du plan étendu $\widehat{\Sigma}$ d'équation $\varphi=l \pi / 2$ (respectivement $\psi=m \pi / 2$ ) avec $l \in \mathbf{Z}$ (respectivement $m \in \mathbf{Z}$ ). La réunion des axes coïncide avec le lieu où la courbure s'annule. En un point d'intersection d'une géodésique $\gamma$ de type $\epsilon= \pm 1$ avec un axe vertical (respectivement horizontal), on constate - vu le choix du vecteur $N$ - que $\epsilon \beta C \geq 0$ (respectivement $\epsilon \beta C \leq 0$ ). Rappelons que les coordonnées $(\varphi, \psi)$ sont monotones le long de $\gamma$. On va voir que l'allure de $\gamma$ dépend essentiellement de la valeur de la constante $C$ qui lui est associée.

Si $C^{2}<1$, la fonction $\beta$ est périodique et prend une infinité de fois les valeurs $\pm C$ et donc ses deux bouts partent à l'infini. De plus, on peut voir que $\beta$ prend deux fois consécutivement la valeur $C$ avant de prendre deux fois consécutivement la valeur $-C$. Ce qui traduit le fait que $\gamma$ coupe deux axes verticaux consécutifs puis deux axes horizontaux consécutifs et ainsi de suite. Ce comportement est illustré par la courbe (a) de la figure 1. Si $C^{2}>1$, à nouveau la fonction $\beta$ est périodique, elle prend une infinité de fois la valeur $C$ ou $-C$ et donc ses deux bouts partent à l'infini. Cette fois la fonction $\beta$ est de signe constant, la géodésique $\gamma$ ne coupe donc que des axes horizontaux ou que des axes verticaux. Ainsi, $\gamma$ est contenue dans une bande horizontale ou verticale de "largeur" $\pi / 2$ bordée par deux axes. De plus, les bouts de $\gamma$ sont asymptotes aux axes. En effet, pour fixer les idées prenons $\gamma=(\varphi, \psi)$ avec $0<\varphi<\pi / 2, \lim _{t \rightarrow \infty} \varphi(t)=\varphi_{0} \leq \pi / 2$ et $\lim _{t \rightarrow \infty} \psi(t)=+\infty$, de sorte que $C>1$, $\epsilon=1$ et $\beta<0$. Le long de $\gamma$, on a

$$
\frac{1}{\delta} \psi^{\prime} \sin (2 \varphi)=C-\beta \quad \text { et } \quad 2 \varphi^{\prime} \psi^{\prime}=\delta
$$


Par suite, si $\varphi_{0}<\pi / 2$, alors $M=\sup \psi^{\prime}<\infty$ ( $\beta$ est bornée), $\delta \geq \delta_{0}>0$ et $\varphi^{\prime} \geq \delta_{0} /(2 M)>0$ quand $t$ tend vers l'infini, ce qui est absurde. Ce comportement est illustré par la courbe (b) de la figure 1.

Si $C^{2}=1$ et $\beta \neq 0$ la courbure ne s'annule que deux fois le long de $\gamma$ qui s'accumule donc aux deux bouts sur un point singulier. La fonction $\beta$ ne change pas de signe et la géodésique est contenue dans un rectangle horizontal ou vertical bordé par des axes, de "largeur" $\pi / 2$ et de "longueur" $3 \pi / 2$. Ce comportement est illustré par la courbe (c) de la figure 1 . Si $C^{2}=1$ et $\beta=0$, le support de $\gamma$ est connu: il s'agit d'un segment diagonal reliant deux singularités (voir la courbe (d) de la figure 1).

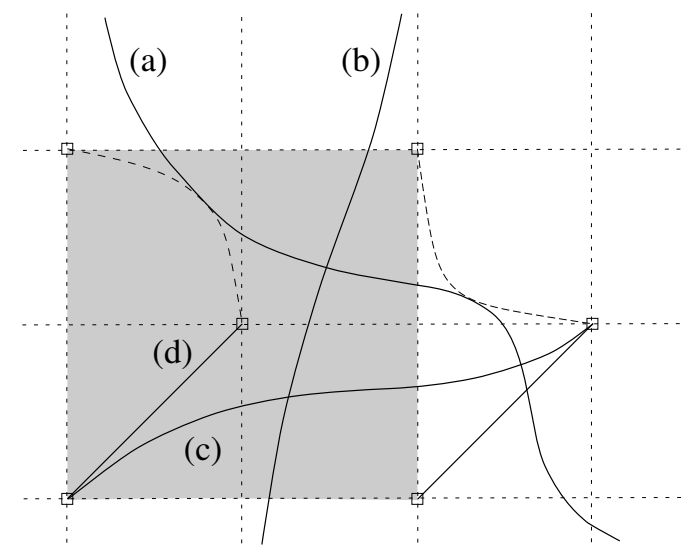

Figure 1: Géodésiques de $\widehat{\Sigma}$ : (a) $C^{2}<1$; (b) $C^{2}>1$; (c) $C^{2}=1, \beta \neq 0 ;$ (d) $C^{2}=1, \beta=0$

Proposition 1.15 Un tore de Clifton-Pohl admet exactement 8 géodésiques fermées (4 de type lumière, 2 de type espace et 2 de type temps) et seulement 4 géodésiques complètes. Les ensembles $\alpha$-limite et $\omega$-limite d'une géodésique sont toujours distincts et constitués d'une seule géodésique fermée.

Preuve Soit $\gamma$ une géodésique du tore de Clifton-Pohl et soit $\widehat{\gamma}$ un relevé de $\gamma$ à $\Sigma_{\mathrm{CP}}$ (qui correspond à la zone grisée de la figure 1). Cette géodésique se prolonge en une géodésique (que l'on note toujours $\widehat{\gamma}$ ) de $\widehat{\Sigma}$. Si $\widehat{\gamma}$ n'est pas contenue dans les diagonales $x^{2}=y^{2}$, elle sort de $\Sigma_{\mathrm{CP}}$ et donc $\gamma$ n'est pas complète. Si $\gamma$ est fermée et de type \pm 1 alors elle est complète. Ce qui prouve que les seules géodésiques fermées de type \pm 1 du tore de Clifton-Pohl sont les 4 obtenues par projection de $x^{2}=y^{2}$. De plus il est bien connu que chaque feuilletage de type lumière du tore de Clifton-Pohl est constitué de deux composantes de Reeb. Il existe donc exactement 4 géodésiques 
fermées de type lumière et toute géodésique de type lumière s'accumule sur deux d'entre elles.

Considérons maintenant une géodésique $\gamma$ de type \pm 1 telle que $\widehat{\gamma}$ n'est pas incluse dans les diagonales $x^{2}=y^{2}$, c'est-à-dire telle que $\widehat{\gamma}$ sort de $\Sigma_{\mathrm{CP}}$. Dans $\Sigma_{\mathrm{CP}}$ en coordonnées $(x, y), \widehat{\gamma}$ part à l'infini en étant asymptote à une droite horizontale ou verticale (géodésique de lumière). Ce bout de $\gamma$ s'accumule sur une géodésique fermée de type lumière. C'est le comportement auquel on assiste en chaque bout si $C^{2} \neq 1$. D'après la discussion précédente, on peut affirmer que les deux géodésiques sur lesquelles s'accumule $\gamma$ appartiennent au même feuilletage de lumière si et seulement si $C^{2}>1$ (voir figure 1 ).

Quand $C^{2}=1$, un bout de $\widehat{\gamma}$ sort de $\Sigma_{\mathrm{CP}}$ et s'accumule sur une géodésique fermée de type lumière, tandis que l'autre bout s'accumule sur une singularité de $\widehat{\Sigma}$ (le 0 de $\Sigma_{\mathrm{CP}}$ ou un point à l'infini). Sans perte de généralité, on peut supposer que $\gamma$ est définie sur $\left[0,+\infty\left[\right.\right.$ et que $\widehat{\gamma}\left(\left[0,+\infty[) \subset V=\left\{(x, y) \in \Sigma_{\mathrm{CP}} ; x>0, y>0\right\}\right.\right.$. On sait (voir proposition 1.10) que $\beta^{\prime}(t)$ tend vers 0 lorsque $t$ tend vers $+\infty$. En utilisant les expressions (7) et (3), on en déduit que $\left(x^{2}-y^{2}\right)\left(x^{2}+y^{2}\right)^{-1}$ tend aussi vers 0 et donc que $x / y$ tend vers 1 . Cela signifie que $\widehat{\gamma}$ coupe toutes les trajectoires de $K$ voisine de la diagonale de $V$ et donc que $\gamma$ s'accumule sur la géodésique fermée obtenue en projetant cette diagonale.

Remarque 1.16 La dynamique de ce flot géodésique est donc très différente de celle d'un flot géodésique riemannien. En un certain sens (que l'on pourrait préciser) ce n'est plus une dynamique conservative.

Remarque 1.17 Le plan de Clifton-Pohl n'est pas le seul ouvert de $\widehat{\Sigma}$ possédant un quotient compact. Ainsi, l'ouvert $W=\{(\varphi, \psi) \in \widehat{\Sigma} ; 0<\psi<\pi / 2\}$ est stable par le groupe $\Gamma$ engendré par le temps 1 du flot de $K$ et la translation de vecteur $(\pi, 0)$. On peut vérifier que $W / \Gamma$ est un tore lorentzien bien différent des tores de Clifton-Pohl. Ce tore possède notamment des points conjugués (par exemple sur la géodésique (a) de la figure 1).

\section{Points conjugués, vus de l'espace des métriques}

\subsection{L'ouvert des métriques à points conjugués}

Dans la suite, l'espace des métriques lorentziennes d'une variété compacte sera muni des topologies $\mathcal{C}^{k}(0 \leq k \leq \infty)$. Le but de ce paragraphe est de montrer que l'ensemble des métriques sur le tore ayant des points conjugués est un ouvert pour la topologie $\mathcal{C}^{2}$ et que les tores de Clifton-Pohl sont dans l'adhérence de cet ensemble. 
Proposition 2.1 Soit $g$ une métrique lorentzienne sur une variété compacte $M$ et soit $v$ vecteur tangent de type temps pour $g$. On suppose que la $g$-géodésique issue de $v$ a des points conjugués. Alors il existe un $\mathcal{C}^{2}$-voisinage $\Omega$ de $g$ dans l'espace des métriques lorentziennes sur $M$ tel que toute métrique $h \in \Omega$ possède des points conjugués le long de la $h$-géodésique issue du vecteur $v$.

Preuve Commençons par remarquer que les géodésiques issues de $v$ associées aux métriques proches de $g$ sont toutes définies sur un même segment et évidemment de type temps.

Affirmation Soit $g$ une métrique lorentzienne sur $M$ compacte, soit $v$ un vecteur de $T M$ et soit $\gamma$ la géodésique issue de $v$. On suppose que $\gamma$ est définie sur un segment $[a, b]$. Si $V$ est un voisinage de $\gamma([a, b])$ dans $T M$ alors il existe $\Omega$ un $\mathcal{C}^{1}$-voisinage de $g$ dans l'espace des métriques lorentziennes sur $M$ tel que pour tout $h \in \Omega$ la $h$-géodésique issue de $v$ est définie sur $[a, b]$ et $\gamma_{h}([a, b]) \subset V$.

Preuve Pour toute métrique $h$ sur $M$ on désigne par $X_{h}$ le générateur du flot géodésique de $h$. L'équation des géodésiques d'une métrique $h$ s'écrivant en fonction des dérivées premières de $h$, on voit que si $\left(h_{n}\right)$ est une suite de métriques lorentziennes qui converge vers $g$ pour la topologie $\mathcal{C}^{1}$ alors $\left(X_{h_{n}}\right)$ converge uniformément sur tout compact vers $X_{g}$. Ainsi, sur tout compact de $T M$, les trajectoires de $X_{h_{n}}$ convergent uniformément vers celles de $X_{g}$. Autrement dit, si on note $\gamma_{n}$ la $h_{n}$-géodésique issue de $v$, pour tout voisinage $V$ de $\gamma([a, b])$, il existe $N \in \mathbf{N}$ tel que pour tout $n>N$, la géodésique $\gamma_{n}$ est définie sur $[a, b]$ et $\gamma_{n}([a, b]) \subset V$. La topologie $\mathcal{C}^{1}$ étant métrisable puisque $M$ est compacte, on en déduit le résultat.

Ce point étant établi la preuve est la même que dans le cas riemannien (voir [16, page 43]). Soit $\gamma$ la géodésique donnée dans l'énoncé de la proposition 2.1. Si $\gamma(a)$ et $\gamma(b)$ sont deux points conjugués alors il existe $\varepsilon>0$ tel que $\gamma$ est définie sur $[a-\varepsilon, b+\varepsilon]$. D'après l'affirmation, il existe un voisinage $\Omega$ de $g$ tel que pour tout $h \in \Omega$ la $h$ géodésique issue de $v$ est définie sur $[a-\varepsilon, b+\varepsilon]$. Ainsi la "forme indice" $I_{[a-\varepsilon, b+\varepsilon]}^{h}$ donnée par

$$
I_{[a-\varepsilon, b+\varepsilon]}^{h}(X, Y)=-\int_{a-\varepsilon}^{b+\varepsilon} h\left(X^{\prime \prime}+R_{h}\left(X, \gamma_{h}^{\prime}\right) \gamma_{h}^{\prime}, Y\right) d t
$$

est bien définie pour toute métrique $h \in \Omega$. La forme $I_{[a-\varepsilon, b+\varepsilon]}^{h}$ est définie négative si et seulement s'il n'existe pas de points conjugués le long de $\gamma_{h}$ entre $\gamma(a-\varepsilon)$ et $\gamma(b+\varepsilon)$ (voir [14, page 274]). Si $h$ est une métrique $\mathcal{C}^{2}$-proche de $g$ alors son tenseur de courbure $R_{h}$ est $\mathcal{C}^{0}$-proche de celui de $g$ et donc $I_{[a-\varepsilon, b+\varepsilon]}^{h}$ est proche 
de $I_{[a-\varepsilon, b+\varepsilon]}^{g}$. Par hypothèse l'indice de $I_{[a-\varepsilon, b+\varepsilon]}^{g}$ est non nul et par semi-continuité inférieure de l'indice on en déduit que cette propriété subsiste sur un $\mathcal{C}^{2}$-voisinage de $g$.

Remarque 2.2 Le fait que les métriques possédant des points conjugués "de type temps" forment un ouvert (en topologie $\mathcal{C}^{2}$ ) se voit aussi aisément à partir de l'indice de Maslov (voir par exemple [13, Section 3] pour cette notion). L'approche avec la forme indice a été privilégiée pour son utilité dans la preuve de la proposition 2.3.

Proposition 2.3 Chaque tore de Clifton-Pohl est limite, pour la topologie $\mathcal{C}^{\infty}$, d'une suite de tores lorentziens possédant des points conjugués.

Preuve Nous nous inspirons du cas riemannien traité dans [16]. Ici, la présence d'une géodésique plongée $\gamma$ avec un champ de Jacobi s'annulant au bord du plan de CliftonPohl permet de conclure plus simplement. On part d'une perturbation de la métrique à support petit coupant $\gamma$ et triviale à l'ordre 1. Cette perturbation est ensuite propagée de façon à passer au quotient, l'existence de points conjugués étant assurée par un contrôle ad hoc de la forme indice.

Rappelons que la métrique de Clifton-Pohl $g_{\mathrm{CP}}=2\left(x^{2}+y^{2}\right)^{-1} d x d y$ est munie du champ de Killing $K=x \partial_{x}+y \partial_{y}$. Notons $V$ le quadrant positif $x>0, y>0$. Soit $\beta_{0}$ la fonction définie dans la proposition 1.10 pour $C=0$ et soit $\omega_{1}$ le quart de période de $\beta_{0}$. Il existe des coordonnées globales $\left.(s, t) \in\right]-\omega_{1}, \omega_{1}[\times \mathbf{R}$ sur $V$ centrées au point $(x, y)=(1,1)$ et telles que

$$
\partial_{s}=K, \quad \partial_{t} \cdot K=0 \quad \text { et } \quad \partial_{t} \cdot \partial_{t}=-1 .
$$

Dans ces coordonnées, la métrique s'écrit $h=\beta_{0}(t)^{2} d s^{2}-d t^{2}$ et la courbe définie par $\gamma(t)=(0, t)(t \in]-\omega_{1}, \omega_{1}[)$ est géodésique. Étant donné $s_{0}>0$, on peut trouver des fonctions $f_{0}$ de norme $\mathcal{C}^{\infty}$ arbitrairement petite, à support dans la boule de centre 0 et de rayon $\min \left(\omega_{1}, s_{0} / 4\right)$ et vérifiant

$f_{0}(0, t)=\partial_{t} f_{0}(0, t)=0, \quad \partial_{s, t}^{2} f_{0}(0, t) \geq 0 \quad$ et $\quad \partial_{s, t}^{2} f_{0}(0,0)>0 \quad(t \in]-\omega_{1}, \omega_{1}[)$.

Notons $f$ la fonction invariante par le temps $s_{0}$ du flot de $K$ obtenue en propageant $f_{0}$ et posons

$$
h_{f}=\beta_{0}^{2} d s^{2}-d t^{2}+2 f d s d t .
$$

Par un calcul direct, on voit que $\gamma$ est une $h_{f}$-géodésique le long de laquelle la courbure de $h_{f}$ vaut $\beta_{0}^{\prime \prime} / \beta_{0}-\partial_{s, t}^{2} f / \beta_{0}^{2}=-2 \beta_{0}^{2}-\partial_{s, t}^{2} f / \beta_{0}^{2}$. Dans les coordonnées initiales $(x, y)$, les métriques précédentes fournissent des perturbations de $g_{\mathrm{CP}}$ invariantes par 
le temps $s_{0}$ du flot de $K$. Elles définissent donc des métriques sur le tore, arbitrairement proches de la métrique d'un tore de Clifton-Pohl donné.

Cela étant, la fonction $\beta_{0}$ définit un champ de Jacobi le long de $\gamma$ qui tend vers 0 aux extrémités de $\gamma$ et qui vérifie $\beta_{0}^{\prime}(0)=0$. Pour tout $\varepsilon>0$, on désigne par $J_{\varepsilon}^{-}$(respectivement $J_{\varepsilon}^{+}$) le champ de Jacobi le long de $\gamma$ qui vérifie $J_{\varepsilon}^{-}(0)=1$ (respectivement $J_{\varepsilon}^{+}(0)=1$ ) et $J_{\varepsilon}^{-\prime}(0)=\varepsilon$ (respectivement $\left.J_{\varepsilon}^{+\prime}(0)=-\varepsilon\right)$. On définit alors la fonction $J_{\varepsilon}$ par $J_{\varepsilon}=J_{\varepsilon}^{-}$à gauche de 0 et $J_{\varepsilon}=J_{\varepsilon}^{+}$à droite de 0 . Il existe des réels $a_{\varepsilon}$ et $b_{\varepsilon}$, tels que $a_{\varepsilon}<0<b_{\varepsilon}$, où la fonction $J_{\varepsilon}$ s'annule. De plus lorsque $\varepsilon$ tend vers 0 ces points s'écartent et tendent vers les bornes de l'intervalle de définition de $\gamma$. Ainsi, on peut supposer que ni $a_{\varepsilon}$ ni $b_{\varepsilon}$ n'appartiennent au support de $f$. On désigne par $I_{\left[a_{\varepsilon}, b_{\varepsilon}\right]}^{f}$ la forme indice associée à la métrique $h_{f}$ et au segment géodésique $\gamma\left(\left[a_{\varepsilon}, b_{\varepsilon}\right]\right)$. Il est maintenant facile de voir que pour $f$ donnée il existe $\varepsilon>0$ tel que

$$
I_{\left[a_{\varepsilon}, b_{\varepsilon}\right]}^{f}\left(J_{\varepsilon}, J_{\varepsilon}\right)>0 .
$$

La forme indice n'étant pas définie négative, il existe des points conjugués pour la métrique $h_{f}$ le long de $\gamma$ (voir à nouveau [14, page 274]).

Remarque 2.4 La méthode précédente fournit également des perturbations $\mathcal{C}^{\infty}$ de toutes les métriques exhibées aux sections 2.3 et 2.4 par des métriques ayant des points conjugués. En effet, il suffit de relever la géodésique $\gamma$ au revêtement universel de $\Sigma_{\mathrm{CP}}$ (voir (16)), puis dans chaque cas de propager une petite perturbation comme ci-dessus par le groupe fondamental.

\subsection{Composantes connexes de l'espace de métriques}

Soit $L$ l'espace des formes quadratiques lorentziennes sur un plan vectoriel réel et soit $L_{+}$, respectivement $L_{*}$, l'espace des formes $q \in L$ munies d'un choix d'une composante du cône négatif $q<0$ (orientation du temps), respectivement du cône positif $q>0$ (orientation de l'espace). Il est clair que

$$
L \simeq \mathbf{G L}_{2}(\mathbf{R}) / \boldsymbol{O}(1,1), \quad L_{+} \simeq \mathbf{G L}_{2}(\mathbf{R}) / \boldsymbol{O}_{+}(1,1) \quad \text { et } \quad L_{*} \simeq \mathbf{G L}_{2}(\mathbf{R}) / \boldsymbol{O}_{*}(1,1),
$$

où $\boldsymbol{O}(1,1)$ est le groupe orthogonal de Lorentz du plan et $\boldsymbol{O}_{+}(1,1)$ (respectivement $\left.\boldsymbol{O}_{*}(1,1)\right)$ son sous-groupe préservant chaque composante du cône négatif (respectivement positif). On sait aussi (voir par exemple [17, Section 40]) que le choix d'une structure euclidienne sur le plan permet de définir - via la décomposition polaire - une rétraction par déformation de $L$ sur un sous-ensemble difféomorphe au projectif $P_{\mathbf{R}}^{1}$. L'espace $L$ a donc le type d'homotopie du cercle $S^{1}$; il en est de même pour $L_{+}$et $L_{*}$ qui sont des revêtements doubles connexes de $L$. 
Soit $\Sigma$ une surface. On notera $L \Sigma, L_{+} \Sigma, L_{*} \Sigma$ les fibrés sur $\Sigma$ associés à $L$, $L_{+}, L_{*}$ et $\mathcal{L}(\Sigma), \mathcal{L}_{+}(\Sigma), \mathcal{L}_{*}(\Sigma)$ les espaces de sections correspondants (métriques lorentziennes sur $\Sigma$ ). Noter que les composantes connexes de $\mathcal{L}(\Sigma)$ coïncident pour toutes les topologies $\mathcal{C}^{k}(k=0, \ldots, \infty)$. Le choix d'une métrique riemannienne auxiliaire sur $\Sigma$ fournit une rétraction par déformation de $L \Sigma$ sur le fibré projectif tangent à $\Sigma$, noté $P \Sigma$. De même $\mathcal{L}(\Sigma)$ a le type d'homotopie de l'espace des champs de droites sur la surface $\Sigma$. Enfin, une métrique lorentzienne est orientable en temps (respectivement en espace) si elle se relève à $L_{+} \Sigma$ (respectivement $L_{*} \Sigma$ ).

Rappelons qu'il existe deux actions naturelles sur les métriques lorentziennes d'une surface: celle des difféomorphismes, en particulier le groupe $\pi_{0} \operatorname{Diff}(\Sigma)$ agit sur les composantes respectives de $\mathcal{L}(\Sigma), \mathcal{L}_{+}(\Sigma)$ et $\mathcal{L}_{*}(\Sigma)$, et l'inversion du signe des métriques qui définit une involution de $\mathcal{L}(\Sigma)$ et échange bijectivement $\mathcal{L}_{+}(\Sigma)$ et $\mathcal{L}_{*}(\Sigma)$.

\subsection{Métriques sans points conjugués sur le tore}

Comme le fibré $L T$ sur le tore $T$ est trivial, l'espace $\mathcal{L}(T)$ des métriques lorentziennes (lisses) sur $T$ s'identifie aux applications (lisses) de $T$ dans $L$. Noter que le groupe $\mathbf{G L}_{2}(\mathbf{Z})$ agit par difféomorphismes sur $T$, donc sur les composantes de $\mathcal{L}(T)$. Étant donnée une métrique lorentzienne sur le tore $T$, on peut choisir un champ de droites négatives $\xi^{-}$et un champ de droites positives $\xi^{+}$. Les champs $\xi^{-}$et $\xi^{+}$sont partout transverses et homotopes; pour cette raison l'inversion de signe des métriques agit trivialement sur les composantes de $\mathcal{L}(T)$. Le 2-tissu isotrope de la métrique définit deux champs de droites (ce qui ne sera plus le cas sur la bouteille de Klein, voir section 2.4) homotopes aux précédents. Ces 4 champs sont simultanément orientables ou non suivant que la métrique se relève ou non au fibré $L_{+} T$.

On sait (voir [4] et [11, II-7]) que l'ensemble des classes d'homotopie d'applications continues de $T$ dans $\boldsymbol{S}^{1}$ s'identifie à $H^{1}(T, \mathbf{Z}) \simeq \mathbf{Z}^{2}$. Pour chaque métrique lorentzienne sur $T$ on choisit l'un des feuilletages de lumière. Celui-ci comprend un nombre fini de composantes de Reeb. Après avoir orienté les feuilles fermées, on note $p_{+}$ (respectivement $p_{-}$) le nombre de ces composantes positives (respectivement négatives) et on pose

$$
n=\left|p_{+}-p_{-}\right| \in \mathbf{N} \text {. }
$$

L'entier $n$ est le nombre minimal de composantes de Reeb d'une métrique de la composante de $\mathcal{L}(T)$ (voir [15] pour les détails). Il caractérise les orbites de $\mathbf{G L}_{2}(\mathbf{Z})$. Les métriques sont temporellement orientables ou non suivant la parité de $n$.

On peut expliciter un revêtement universel des tores de Clifton-Pohl en paramétrant les méridiens (courbes orthogonales au champ de Killing) et les parallèles (trajectoires 
du champ de Killing). Par exemple, en posant $x=\exp (u) \cos (\theta), y=\exp (u) \sin (\theta)$ pour $(x, y) \in \Sigma_{\mathrm{CP}}$ (voir section 1.2) on trouve la métrique

$$
\sin 2 \theta\left(d u^{2}-d \theta^{2}\right)+2 \cos 2 \theta d u d \theta, \quad(u, \theta) \in \mathbf{R}^{2},
$$

sans points conjugués (théorème 2). Ce plan lorentzien sera noté $\widetilde{\Sigma}_{\mathrm{CP}}$. Les directions des coordonnées correspondent aux méridiens $(u=$ cte) et aux parallèles $(\theta=$ cte). Les feuilletages de lumière $\mathcal{F}_{i}(i=1,2)$ se décomposent en composantes de Reeb: il s'agit de bandes horizontales de largeur $\pi$, d'âmes $\theta=k \pi(k \in \mathbf{Z})$ pour l'un des feuilletages et $\theta=\pi / 2+k \pi(k \in \mathbf{Z})$ pour l'autre. Nous supposerons que les $\mathcal{F}_{i}$ sont orientés.

Revenons aux composantes de $\mathcal{L}(T)$. Le cas $n=0$ (orbite "triviale" de $\mathbf{G L}_{2}(\mathbf{Z})$ ) correspond à une seule composante contenant les métriques plates qui sont évidemment sans points conjugués. Ensuite, soit $T_{n}(n \geq 1)$ le quotient de $\widetilde{\Sigma}_{\mathrm{CP}}$ par les translations $t$ et $\tau^{n}$ avec

$$
t(u, \theta)=(u+1, \theta) \quad \text { et } \quad \tau(u, \theta)=(u, \theta+\pi), \quad(u, \theta) \in \mathbf{R}^{2} .
$$

Alors $T_{n}$ est un revêtement cyclique de degré $n$ de $T_{1}$ (en particulier $T_{2}$ est un tore de Clifton-Pohl) et c'est un tore lorentzien sans points conjugués, orientable pour $n$ pair. Cette suite $T_{n}$ représente toutes les orbites de $\mathbf{G L}_{2}(\mathbf{Z})$, excepté l'orbite triviale (composante des métriques plates). En faisant agir $\mathbf{G L}_{2}(\mathbf{Z})$, on obtient donc une métrique sans points conjugués dans chaque composante connexe de $\mathcal{L}(T)$. Cela prouve la première partie du théorème 1 .

\subsection{Métriques sans points conjugués sur la bouteille de Klein}

Soit $K$ la bouteille de Klein, présentée comme suspension d'une isométrie indirecte d'un cercle euclidien (deux points fixes et renverse l'orientation). Ainsi $K$ est décomposée en deux rubans de Möbius (dont les âmes correspondent aux points fixes) et feuilletée par les méridiens (cercles de la suspension). On fixe un méridien (orienté) $\beta$ ainsi que l'une des âmes $\alpha$ de $K$. D'après l'appendice, tout champ de droites $\xi$ sur $K$ est caractérisé à homotopie près par un élément $\left(n_{\xi}, \bar{m}_{\xi}\right) \in \mathbf{Z} \oplus \mathbf{Z} / 2 \mathbf{Z}$ défini par ses restrictions à $\beta$ et à $\alpha$. De plus, il résulte de cette étude que $\bar{m}_{\xi}=\bar{m}_{\xi^{\prime}}$ si et seulement si $\xi$ et $\xi^{\prime}$ se coupent homotopiquement au-dessus de $\alpha$ (donc deux champs partout transverses ne sont jamais homotopes) et qu'il n'existe pas trois champs de droites mutuellement transverses en tout point de $K$. Par suite, le 2-tissu isotrope d'une métrique lorentzienne sur $K$ ne définit jamais de champs de droites. Chaque composante connexe de $\mathcal{L}(K)$ sera donc caractérisée par l'élément $(n, \bar{m}) \in \mathbf{Z} \oplus \mathbf{Z} / 2 \mathbf{Z}$ associé à un champ de droites négatives $\xi^{-}$. 
Il est connu que $\pi_{0} \operatorname{Diff}(K)$ est isomorphe à $\mathbf{Z} / 2 \mathbf{Z} \oplus \mathbf{Z} / 2 \mathbf{Z}$ (voir par exemple [12, page 315]). Considérons une symétrie $\sigma_{1}$ par rapport à un méridien, une symétrie centrale $\sigma_{2}$ centrée sur le bord commun des deux rubans de Möbius et la symétrie $\sigma_{3}$ par rapport à ce bord. L'action de ces difféomorphismes sur les classes d'homotopie de champs de droites, c'est-à-dire sur les composantes connexes de $\mathcal{L}(K)$, est alors clairement la suivante (voir Remarque A.1):

$$
\left\{\begin{array}{l}
\sigma_{1}(n, \bar{m})=(-n, \bar{m}), \\
\sigma_{2}(n, \bar{m})=(-n, \overline{m+n}), \quad(n, \bar{m}) \in \mathbf{Z} \oplus \mathbf{Z} / 2 \mathbf{Z} . \\
\sigma_{3}(n, \bar{m})=(n, \overline{m+n}),
\end{array}\right.
$$

Cela montre en particulier que les $\sigma_{i}(i=1,2,3)$ représentent avec l'identité tous les éléments de $\pi_{0} \operatorname{Diff}(K)$. L'inversion du signe de la métrique correspond quant à elle à

$$
\iota(n, \bar{m})=(n, \overline{m+1}), \quad(n, \bar{m}) \in \mathbf{Z} \oplus \mathbf{Z} / 2 \mathbf{Z} .
$$

Il s'agit de l'opération qui à tout champ de droites associe l'unique champ (à homotopie près) qui lui est transverse.

Ensuite, un champ de droites $\xi$ est orientable si et seulement si $n$ est pair et $\xi_{\mid \alpha}$ est homotope au champ des tangentes à l'âme $\alpha(\bar{m}=\overline{0}$ suivant l'appendice), il en est alors de même pour l'autre âme, voir Remarque A.1. Une métrique lorentzienne $g \in \mathcal{L}(K)$ ne sera donc orientable ni en temps ni en espace pour $n$ impair; si $n$ est pair, $g$ sera orientable en espace ou en temps selon que les âmes sont tranverses (à homotopie près) à $\xi^{-}$ou non.

Le plan $\widetilde{\Sigma}_{\mathrm{CP}}$ permet également de construire des métriques sans points conjugués sur la bouteille de Klein $K$. Posons

$$
\sigma(u, \theta)=(u+1, \pi / 2-\theta), \quad(u, \theta) \in \mathbf{R}^{2},
$$

et notons $K_{n}$ le quotient de $\widetilde{\Sigma}_{\mathrm{CP}}$ par le sous-groupe $\left\langle\sigma, \tau^{n}\right\rangle(n \geq 1)$. Remarquer que les âmes correspondent à $\theta=\pi / 4$ modulo $n \pi / 2$, donc sont de type $(-1)^{n}$. L'entier $n$ est le nombre de composantes de Reeb d'un champ de droites négatives. Pour $n$ pair, $K_{n}$ est orientable en espace (mais pas en temps), et $K_{n}$ n'est orientable ni en temps ni en espace pour $n$ impair. La composante connexe de $K_{n}$ correspond à $(n, \overline{1}) \in \mathbf{Z} \oplus \mathbf{Z} / 2 \mathbf{Z}$. L'orbite de $K_{n}$ sous l'action de $\pi_{0} \operatorname{Diff}(K)$ a 4 éléments pour $n$ impair et 2 éléments pour $n$ pair; dans ce dernier cas, les métriques $-K_{n}$ et $\sigma_{1}^{*}\left(-K_{n}\right)$ représentent les deux composantes avec la même valeur de $|n|$ et orientables en temps. Si l'on ajoute à cette liste les deux métriques plates sur $K$ (associées à $n=0$ ), on obtient une métrique sans points conjugués dans chaque composante connexe de $\mathcal{L}(K)$. Ce qui achève la preuve du théorème 1. 


\section{Appendice: champs de droites sur la bouteille de Klein}

Soit $K$ la bouteille de Klein et soit $P K$ le fibré projectif associé au fibré tangent de $K$. La suite exacte d'homotopie de ce fibré s'écrit

$$
0 \longrightarrow \mathbf{Z} \longrightarrow \pi_{1}(P K) \longrightarrow \pi_{1}(K) \longrightarrow 1 .
$$

Notons $\underline{\mathbf{Z}}$ le $\pi_{1}(K)$-module défini par cette suite exacte. L'énoncé suivant est naturel.

Théorème L'ensemble des composantes connexes de l'espace des champs de droites sur la bouteille de Klein s'identifie avec $H^{1}\left(\pi_{1}(K), \underline{\mathbf{Z}}\right) \simeq \mathbf{Z} \oplus \mathbf{Z} / 2 \mathbf{Z}$.

Preuve Tout d'abord, il est immédiat que $H^{1}\left(\pi_{1}(K), \underline{\mathbf{Z}}\right) \simeq \mathbf{Z} \oplus \mathbf{Z} / 2 \mathbf{Z}$. Ce groupe correspond aux scindements de la suite exacte (17), modulo conjugaison par un élément du noyau, ou encore aux dérivations $d: \pi_{1}(K) \rightarrow \underline{\mathbf{Z}}$, modulo les dérivations principales [3, page 89]. Le groupe $\pi_{1}(K)=\left\langle a, b \mid a b a^{-1}=b^{-1}\right\rangle$ agit par $a \cdot k=-k$ et $b \cdot k=k$ $(k \in \mathbf{Z})$. Toute dérivation $d$ est alors déterminée par $(n, m)=(d b, d a) \in \mathbf{Z} \oplus \mathbf{Z}$, les dérivations principales étant caractérisées par $n=0$ et $m \in 2 \mathbf{Z}$.

Ensuite, toute classe d'homotopie de sections du fibré $P K \rightarrow K$ induit évidemment via la suite exacte (17) un élément de $H^{1}\left(\pi_{1}(K), \underline{\mathbf{Z}}\right)$. Cette correspondance peut être explicitée par le comportement des sections au-dessus d'un système générateur de $\pi_{1}(K)$. Soient $\alpha, \beta: \mathbf{R} / \mathbf{Z} \rightarrow K$ une âme et un méridien de $K$, paramétrés de sorte que $\alpha(0)=\beta(0)$ et soit $\gamma: \mathbf{R} / \mathbf{Z} \rightarrow P K$ la fibre paramétrée au-dessus de ce point, noté $p$. On prend comme points bases $p \in K$ et $q=\gamma(0) \in P K$, puis on choisit des revêtements universels pointés $(\widetilde{K}, \widetilde{p})$ et $(\widetilde{P K}, \widetilde{q})$. Enfin, on note $\widetilde{\alpha}, \widetilde{\beta}: \mathbf{R} \rightarrow \widetilde{K}$ et $\widetilde{\gamma}: \mathbf{R} \rightarrow \widetilde{P K}$ les relevés tels que $\widetilde{\alpha}(0)=\widetilde{\beta}(0)=\widetilde{p}$ et $\widetilde{\gamma}(0)=\widetilde{q}$.

Soit maintenant $\xi$ une section de $P K \rightarrow K$. Quitte à "homotoper", on peut supposer que $\xi(p)=q$. La section $\xi$ se relève de façon unique en $\widetilde{\xi}: \widetilde{K} \rightarrow \widetilde{P K}$ tel que $\widetilde{\xi}(\widetilde{p})=\widetilde{q}$. Il existe alors un unique couple $(n, m) \in \mathbf{Z} \oplus \mathbf{Z}$ tel que

$$
\widetilde{\xi} \circ \widetilde{\alpha}(1)=\widetilde{\gamma}(m) \text { et } \widetilde{\xi} \circ \widetilde{\beta}(1)=\widetilde{\gamma}(n),
$$

couple qui correspond à la dérivation $\pi_{1}(K) \rightarrow \underline{\mathbf{Z}}$ associée à $\xi$. Soit [ $\xi$ ] la classe d'homotopie de $\xi$ et soit $\bar{m}$ la classe de $m$ modulo 2 . En explicitant des modèles pour les revêtements universels (voir ci-dessous), on vérifie facilement que la correspondance $[\xi] \mapsto(n, \bar{m}) \in \mathbf{Z} \oplus \mathbf{Z} / 2 \mathbf{Z}$ est bijective.

Pour fixer les idées, on peut prendre $\widetilde{K}=\mathbf{C}$ et $\widetilde{P K}=\widetilde{K} \times \mathbf{R}$ pointé en $(0,0) \in \mathbf{C} \times \mathbf{R}$, l'action de $\pi_{1}(P K)$ étant définie par $\widehat{a} \cdot(z, t)=(\bar{z}+1,-t), \widehat{b} \cdot(z, t)=(z+i, t)$ et $\widehat{c} \cdot(z, t)=(z, t+1)$ pour $(z, t) \in \widetilde{P K}$, où $\widehat{c}$ est un générateur du noyau de (17). Les 
âmes de $K$ correspondent à $\operatorname{Im} z \in \frac{1}{2} \mathbf{Z}$. Un représentant $\xi_{n, m}$ de chaque classe de champ de droites sur $K$ est alors donné par

$$
\widetilde{\xi}_{n, m}(z)=\left(z, n \operatorname{Im} z+\frac{m}{2}\right) \quad(z \in \mathbf{C}) .
$$

Le couple $(n, \bar{m})$ défini par (18) s'interprète comme suit. Tout d'abord, le fibré $P K_{\mid \beta}$ est trivial et l'entier $n$ correspond au degré de l'application de $\beta$ dans le cercle définie par $\xi_{\mid \beta}$. Ensuite, au-dessus de l'âme $\alpha$, le fibré induit $P K_{\mid \alpha}$ est une bouteille de Klein et $\xi_{\mid \alpha}$ est homotope à l'une des deux âmes de $P K_{\mid \alpha}$ selon la parité de $m$. On peut convenir que $\bar{m}=\overline{1}$ quand $\xi_{\mid \alpha}$ est partout transverse à $\alpha$, à homotopie près (ce sera le cas pour le modèle précédent si l'on suppose de plus que la section nulle $\mathbf{C} \times\{0\}$ se projette sur le champ de droites "horizontal" de $K$ ).

Remarque A.1 De façon analogue, la section $\xi$ définit un élément $\bar{m}^{\prime} \in \mathbf{Z} / 2 \mathbf{Z}$ associé à l'autre âme de $K$ et on a la relation $\bar{m}+\bar{m}^{\prime}+\bar{n}=\overline{0}$ ( $n$ comme ci-dessus).

Remerciements Nous remercions le rapporteur de l'article pour ses remarques ainsi que les éditeurs du journal pour leurs commentaires.

\section{Bibliographie}

[1] L Andersson, M Dahl, R Howard, Boundary and lens rigidity of Lorentzian surfaces, Trans. Amer. Math. Soc. 348 (1996) 2307-2329 MR1363008

[2] C Boubel, P Mounoud, C Tarquini, Lorentzian foliations on 3-manifolds, Ergodic Theory Dynam. Systems 26 (2006) 1339-1362 MR2266364

[3] K S Brown, Cohomology of groups, Graduate Texts in Math. 87, Springer, New York (1982) MR672956

[4] N Bruschlinsky, Stetige Abbildungen und Bettische Gruppen der Dimensionszahlen 1 und 3, Math. Ann. 109 (1934) 525-537 MR1512908

[5] D Burago, S Ivanov, Riemannian tori without conjugate points are flat, Geom. Funct. Anal. 4 (1994) 259-269 MR1274115

[6] Y Carrière, L Rozoy, Complétude des métriques lorentziennes de $\boldsymbol{T}^{2}$ et difféormorphismes du cercle, Bol. Soc. Brasil. Mat. 25 (1994) 223-235 MR1306563

[7] L Green, R Gulliver, Planes without conjugate points, J. Differential Geom. 22 (1985) 43-47 MR826423

[8] M Gutiérrez, F J Palomo, A Romero, Lorentzian manifolds with no null conjugate points, Math. Proc. Cambridge Philos. Soc. 137 (2004) 363-375 MR2092065 
[9] G H Halphen, Traité des fonctions elliptiques et de leurs applications. I, GauthierVillars, Paris (1886)

[10] E Hopf, Closed surfaces without conjugate points, Proc. Nat. Acad. Sci. U. S. A. 34 (1948) 47-51 MR0023591

[11] S-t Hu, Homotopy theory, Pure and Applied Mathematics 8, Academic Press, New York (1959) MR0106454

[12] W B R Lickorish, Homeomorphisms of non-orientable two-manifolds, Proc. Cambridge Philos. Soc. 59 (1963) 307-317 MR0145498

[13] F Mercuri, P Piccione, D V Tausk, Stability of the conjugate index, degenerate conjugate points and the Maslov index in semi-Riemannian geometry, Pacific J. Math. 206 (2002) 375-400 MR1926783

[14] B O'Neill, Semi-Riemannian geometry, with applications to relativity, Pure and Applied Math. 103, Academic Press, New York (1983) MR719023

[15] B L Reinhart, Line elements on the torus, Amer. J. Math. 81 (1959) 617-631 MR0111050

[16] RO Ruggiero, On the creation of conjugate points, Math. Z. 208 (1991) 41-55 MR1125731

[17] N Steenrod, The Topology of Fibre Bundles, Princeton Math. Series 14, Princeton Univ. Press (1951) MR0039258

Institut de Mathématiques de Bordeaux, Université Bordeaux 1

351 cours de la Libération, 33405 Talence, France

Christophe.Bavard@math.u-bordeaux1.fr, pierre.mounoud@math.u-bordeaux1.fr

Proposed: Jean-Pierre Otal

Seconded: Yasha Eliashberg, Dmitri Burago

Received: 30 May 2011

Revised: 8 October 2012 